\title{
Impact of flanking chromosomal sequences on localization and silencing by the human non-coding RNA XIST
}

Angela D. Kelsey ${ }^{1 \dagger}$, Christine Yang ${ }^{1 \dagger}$, Danny Leung ${ }^{2,3}$, Jakub Minks ${ }^{1}$, Thomas Dixon-McDougall ${ }^{1}$, Sarah E.L. Baldry ${ }^{1}$, Aaron B. Bogutz ${ }^{1}$, Louis Lefebvre ${ }^{1}$ and Carolyn J. Brown ${ }^{1 *}$ (D)

\begin{abstract}
Background: X-chromosome inactivation is a striking example of epigenetic silencing in which expression of the long non-coding RNA XIST initiates the heterochromatinization and silencing of one of the pair of X chromosomes in mammalian females. To understand how the RNA can establish silencing across millions of basepairs of DNA we have modelled the process by inducing expression of XIST from nine different locations in human HT1080 cells.

Results: Localization of XIST, depletion of Cot-1 RNA, perinuclear localization, and ubiquitination of H2A occurs at all sites examined, while recruitment of $\mathrm{H} 3 \mathrm{~K} 9 \mathrm{me} 3$ was not observed. Recruitment of the heterochromatic features SMCHD1, macroH2A, H3K27me3, and H4K20me1 occurs independently of each other in an integration site-dependent manner. Silencing of flanking reporter genes occurs at all sites, but the spread of silencing to flanking endogenous human genes is variable in extent of silencing as well as extent of spread, with silencing able to skip regions. The spread of H3K27me3 and loss of H3K27ac correlates with the pre-existing levels of the modifications, and overall the extent of silencing correlates with the ability to recruit additional heterochromatic features.

Conclusions: The non-coding RNA XIST functions as a cis-acting silencer when expressed from nine different locations throughout the genome. A hierarchy among the features of heterochromatin reveals the importance of interaction with the local chromatin neighborhood for optimal spread of silencing, as well as the independent yet cooperative nature of the establishment of heterochromatin by the non-coding XIST RNA.
\end{abstract}

Keywords: XIST, Long non-coding RNA, Dosage compensation, X-chromosome inactivation, Nucleolar localization, Facultative heterochromatin, SMCHD1, macroH2A, H3K27me3, H4K20me1

\section{Background}

To avoid a functional gene dosage imbalance between the sexes, one of the two X chromosomes in female placental mammals is transcriptionally silenced [1]. This process of $\mathrm{X}$-chromosome inactivation (XCI) occurs early in development and is generally random in all human tissues with either the paternal or maternal X chromosome becoming the inactive $\mathrm{X}(\mathrm{Xi})$. The $\mathrm{X}$-inactivation centre $(X I C)$, which is located at Xq13 in humans, is the region of the $\mathrm{X}$ that is necessary for the chromosome to be inactivated, and contains the XIST gene (X-inactive specific

\footnotetext{
* Correspondence: Carolyn.Brown@UBC.Ca

${ }^{\dagger}$ Equal contributors

'Department of Medical Genetics, Molecular Epigenetics Group, Life Sciences Institute, University of British Columbia, Vancouver, Canada

Full list of author information is available at the end of the article
}

transcript) [2-4]. Remarkably, the approximately $17 \mathrm{~kb}$ spliced and polyadenylated long non-coding XIST RNA uniquely localizes to the chromosome from which it is transcribed [5]. The coating of the Xi by the XIST RNA results in a substantial epigenetic transformation, losing epigenetic modifications associated with active chromatin (notably histone acetylation) and gaining modifications associated with inactive chromatin (including H3K27me3, H3K9me2/3, H4K20me1, and H2AK119u1). The Xi also becomes enriched in several other proteins, including the histone variant macrohistone $\mathrm{H} 2 \mathrm{~A}$ (macroH2A), the nuclear matrix protein hnRNPU, and the epigenetic regulators SMCHD1 and ASH2L ([6, 7], reviewed in [8]). In addition, the $\mathrm{Xi}$ is peripherally or perinucleolarly localized [9] with perinucleolar targeting during $S$ phase suggested 
to be important for maintenance of silencing. A further feature of the $\mathrm{Xi}$ is silencing of repetitive elements, as visualized by loss of RNA hybridization with a Cot-1 probe for repetitive DNA [10] resulting in what has been termed a 'Cot-1 hole'.

The timing of acquisition of these features has been best studied in mouse, where the differentiation of embryonic stem cells (ESCs) provides an in vitro model for the events of XCI (reviewed in [11]). Early studies in mouse suggested the presence of a developmental window beyond which Xist was unable to induce X-chromosome inactivation [12], although macroH2A could be recruited [13]. After this stage, Xist expression was no longer required for maintenance of silencing [14], consistent with studies in human showing maintenance of silencing in the absence of XIST $[15,16]$. SATB1 has been suggested to be involved in defining such a window for Xist function [17]; however, Satb1/ Satb2-deficient mice are able to undergo X inactivation [18]. More recent studies have shown that induction of Xist can recruit H3K27me3 in mouse somatic cells [19], and XIST induction recruits multiple features of the $\mathrm{Xi}$ in human somatic cells $[10,20]$. In addition, an ongoing role for Xist in stable silencing of the Xi has been shown by loss of Xist resulting in gene reactivation, loss of perinucleolar association and loss of H3K27me3 [21]. Loss of X-linked gene silencing is enhanced by disruption of DNA methylation and other pathways that cooperate with Xist, thus reactivation of $\mathrm{X}$-linked markers has been used to identify additional players in the pathway [22-24]. Characterization of the ongoing role for XIST in somatic cells has important implications for disease, as highlighted by a recent study showing that deletion of Xist results in hematological malignancies in female mice due to reactivation of X-linked genes [25].

There is substantial evidence for cooperativity of multiple silencing pathways in the initial silencing of the chromosome, with XCI able to proceed in the absence of key components of the silencing machinery such as PRC2 [26-28], PRC1 [29], or macroH2A [30]. In addition to multiple factors cooperating in the process of XCI, different X-linked genes may be silenced (or maintained silent) by different players. For example, mutation of the Smchd1 gene results in loss of DNA methylation and partial to full reactivation of approximately $20 \%$ of the X-linked genes in mouse [31, 32]. Surprisingly, many of the marks of an Xi can be recruited by a mouse transgene containing a deletion that makes the Xist RNA defective in silencing, although often the recruitment is not as effective as seen with a full-length Xist (reviewed in [8]). This silencing defective Xist RNA is also able to form a Cot-1 hole [33], consistent with the Cot1 hole not reflecting X-linked gene silencing [34], but rather a core of silenced non-coding DNA [35].

Spread of silencing to autosomal genes has been observed in unbalanced X/autosome translocations; however, the extent of autosomal silencing is highly variable in both humans (reviewed in [36]) and mice [37]. Silencing of autosomal genes has also been observed upon integration of Xist/ XIST transgenes into autosomes, and localization of the RNA to the autosome is able to induce many features of the $\mathrm{Xi}$ including nucleolar localization [10, 20, 21, 38]. Recently, an XIST transgene was integrated into chromosome 21 in induced pluripotent stem cells from an individual with Down's syndrome and corrected gene expression from chromosome 21 to near normal disomic levels [39]. Together, these studies demonstrate the XIST RNA is able not only to spread along autosomal material but also to recruit some of the heterochromatic features associated with XCI to autosomes. The silencing of the trisomic chromosome 21 in induced pluripotent stem cells was proposed as a first step towards 'chromosome therapy' [39], and for such uses of XIST a better understanding of the influence of the chromatin neighborhood is necessary.

XIST expression is required to induce the cascade of changes that cooperatively silence the $\mathrm{X}$, but relatively little is known about the process by which the non-coding RNA recruits these changes. In human somatic cells we have reported the recruitment of several features of XCI, including gene silencing, following XIST expression from an inducible transgene [20,40]. The separation of XIST expression from the myriad of changes that occur during differentiation provides an opportunity to dissect the role of XIST in XCI. By using this inducible XIST transgene to examine the influence of XIST expression induced from nine different integration sites we aimed to establish a hierarchy to the features that are recruited by XIST and determine if any were influenced by the genomic context of the XIST integration. We were able to identify features (Cot-1 hole formation, perinucleolar localization, proximal reporter silencing, and H2AK119u1) that are recruited to all integrations and thus are compatible with any of the sites/genomic contexts tested, a feature that is not recruited to any site examined (H3K9me3) while another set of features (macroH2A, H3K27me3, SMCHD1, and H4K20me1 recruitment) are dependent on genomic context, but appear independent of each other.

\section{Results}

XIST RNA expression results in depletion of Cot-1 RNA and increased perinucleolar location

In the HT1080 cells an inducible XIST cDNA has been previously reported to localize in cis to the chromosome from which it is transcribed, resulting in the recruitment of some chromatin modifications and repression of both flanking reporter genes as well as flanking endogenous genes $[20,40]$. To test whether there are differences in the functionality of the XIST RNA due to influences of local DNA sequences and chromatin environment we integrated a single copy of the full-length inducible XIST cDNA into nine different FRT sites in HT1080 cells 
containing a constitutive Tet-repressor transgene to allow induction of XIST by treatment of the cells with the tetracycline analog doxycycline (DOX). The sites of the FRT integrations were identified by inverse PCR and sequencing to be $1 \mathrm{p}, 3 \mathrm{q}, 4 \mathrm{q}, 7 \mathrm{p}, 7 \mathrm{q}, 8 \mathrm{p}, 12 \mathrm{q}, 15 \mathrm{q}$, and an X chromosome FRT site at Xq23 was previously reported [41]. The integration sites (described in Additional file 1) are in both G-dark and G-light regions (four and five, respectively), and three integrations occurred within a gene.

For each clone, the inducible promoter was activated for 5 days and the localization of XIST RNA assessed by RNA FISH. At each integration site the XIST RNA was able to localize and formed an XIST cloud comparable to that observed in normal female cells (Fig. 1). The level of XIST RNA varied between cell lines and also within different cultures of the same cell line, showing from five- to 30-fold induction of XIST after 5 days of DOX (Additional file 1). The integrations into $3 \mathrm{q}, 7 \mathrm{p}$, and $15 \mathrm{q}$ (all G-dark) showed lower expression by q-RT-PCR, which is consistent with a significantly smaller signal for XIST for the $3 q$ integration clone relative to all other integrations except for $15 \mathrm{q}(P \leq 0.01)$. Co-hybridization with fluorescently labelled Cot-1 also showed depletion of Cot-1 hybridization coincident with the XIST cloud at each integration site, observable in the line diagrams of signal intensity across the XIST cloud (Fig. 1). Recently stable Cot-1 repeat RNA has been shown to be associated with euchromatic chromosomes, yet excluded from the Xi resulting in the Cot-1 RNA hole [42]. We noted differences in the intensity of the Cot-1 holes; however, in attempting to quantify such differences it became apparent that the XIST RNA signal was often
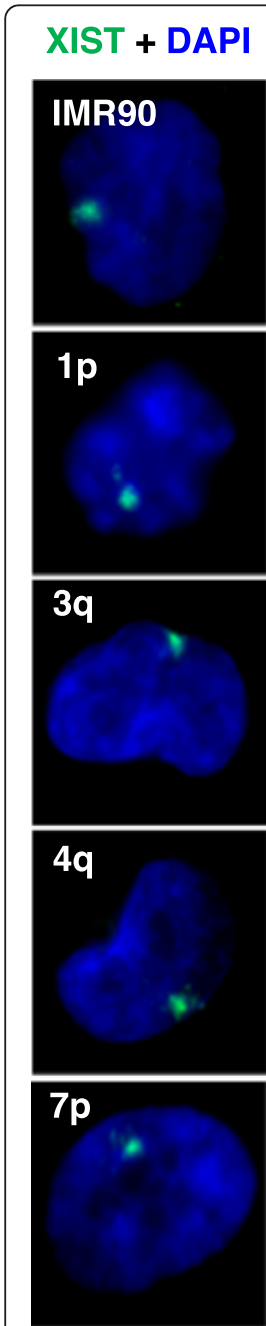

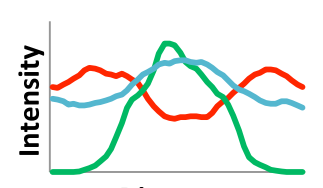

Distance
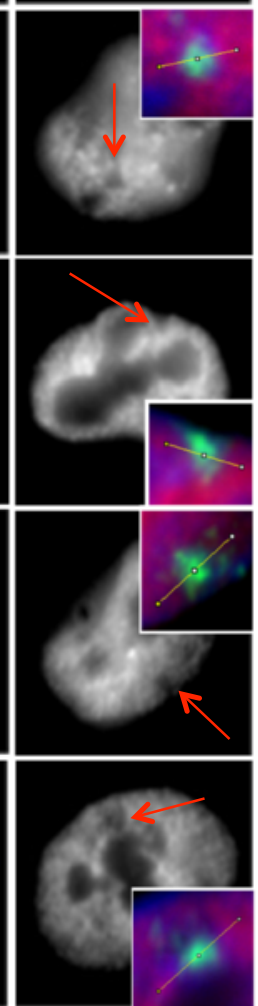

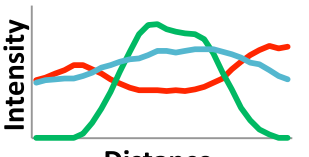

Distance

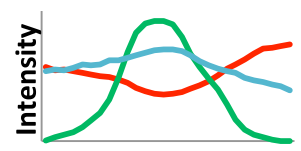

Distance

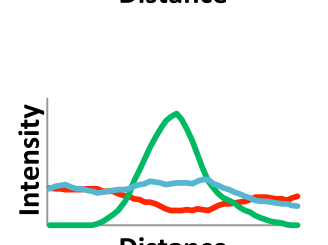

Distance



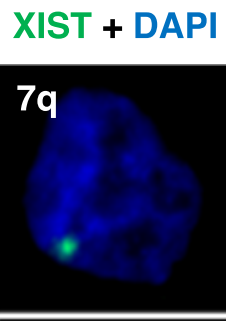
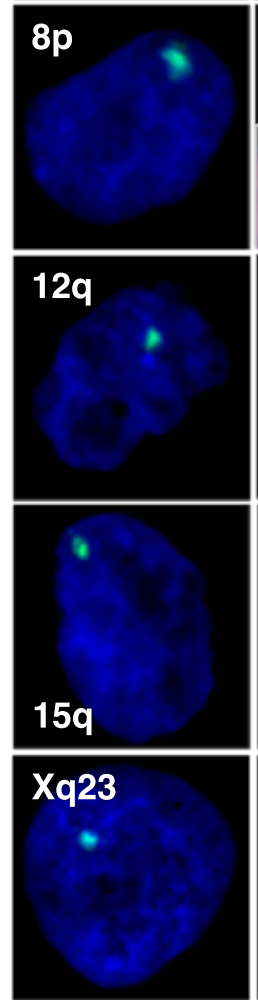

Cot-1
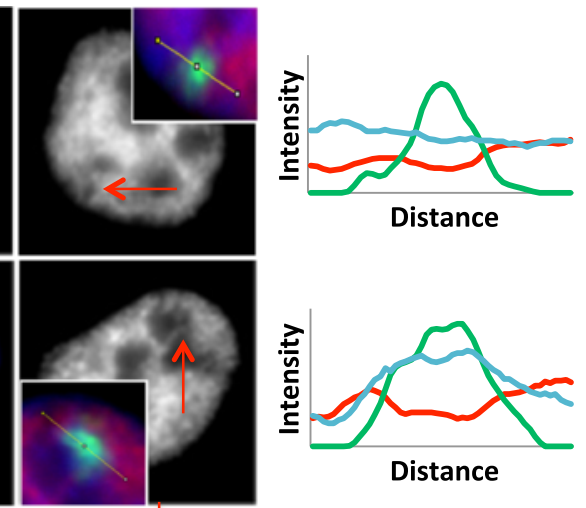

Distance


Distance

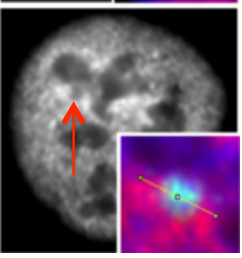

Fig. 1 XIST RNA localizes and forms Cot-1 holes when expressed from nine different integration sites. Shown is XIST RNA FISH (green) upon expression of an inducible XIST transgene integrated into the indicated chromosomal locations in HT1080 cells. IMR90 cells (a female fibroblast line) are shown as a positive comparator. Cells were counter-stained with DAPI (blue) and co-hybridized with Cot-1 (labelled with spectrum-red, but shown in grayscale). Arrow indicates the location of the XIST signal and reduction in Cot-1 staining. Graphs to the right show the RGB intensities across the lines shown in the picture inserts drawn through the XIST clouds (XIST (green), Cot-1 (red), and DAPI (blue)) 
at the nuclear or nucleolar periphery in the HT1080 cells, and that measuring the intensity of the Cot-1 hole could be influenced by nuclear location. The Xi is generally located at the nuclear or nucleolar periphery; however, as these were autosomal integration sites, it seemed that expression of XIST might be altering nuclear location.

To test whether XIST expression was relocating the chromosome from which it was expressed, the XIST signal was scored for being in contact with either the nuclear periphery or the nucleolus, prior to and after XIST induction. Prior to DOX induction there was only a small focus of XIST expression (see [20]); however this signal was sufficient to identify the location of the integrated XIST. In six of the nine integration sites, induction with DOX resulted in a significant increase in perinucleolar localization $(P \leq 0.05)$, a trend shared with the other integration sites (Table 1). Perinuclear association, on the other hand, showed no significant difference for five of the integrations, with three integrations showing a significant increase and the $8 \mathrm{p}$ integration showing a significant decrease $(P \leq 0.01$; Table 1$)$. The full distribution of localization before and after induction of XIST is shown in Additional file 2. The 8p integration site showed the highest proportion of perinucleolarassociated XIST signals (56 \%) and also the greatest increase in perinucleolar association following XIST induction $(27 \%)$. In mouse, localization of Xist to the perinucleolar compartment was shown to be necessary for the silencing activity of Xist [21], leading us to question whether the differing nucleolar recruitment and Cot-1 hole formation that we observed might be reflective of silencing ability.

\section{XIST RNA expression silences nearby reporter and endogenous genes}

We previously reported silencing of a flanking EGFP reporter gene at the $3 \mathrm{q}$ integration site $[20,40]$. At the other FRT sites we did not co-integrate a reporter construct; however, the integration of XIST into the FRT site results in expression of an upstream Hygromycin $(H y g)$ gene. Robust silencing of $H y g$ was observed at all integrations after 5 days of XIST expression (Fig. 2a), suggesting that XIST is able to silence a virally-derived promoter (SV40), consistent with our previous demonstration that an EGFP reporter driven by the CMV promoter could be silenced [20]. The 7q integration site showed significantly less silencing than the $1 p, 3 q, 7 p$, $8 \mathrm{p}, 15 \mathrm{q}$, and $\mathrm{Xq}$ integration sites $(P \leq 0.01)$. As only the repeat A region of XIST is required for silencing of the flanking reporter genes [40], we generated a construct containing the XIST repeat A and a DsRed reporter driven by the mouse Pgk1 gene promoter, which is normally X-linked and subject to XCI (Fig. 2b). We integrated this construct into six of the integration sites and again observed consistent silencing of greater than $90 \%$ (Fig. 2c), suggesting both viral and mouse-derived promoters could be silenced by XIST in the HT1080 cells. Consistent with previous results with the EGFP reporter gene at 3q, silencing of the dsRed reporter was reversible when induction of XIST expression was stopped by removal of DOX (Fig. 2d).

Given the capacity of the XIST RNA to silence in cis, and the apparent spread of the RNA along the chromosome based on our RNA FISH data, we questioned whether there would be silencing of endogenous genes at additional sites adjacent to the XIST transgenes. The HT1080 cells remain diploid although they carry several structural rearrangements $(46, \mathrm{XY}$,del $(1)(\mathrm{p} 21)$, i(3)(p10), i(3)(q10), der(4)t(1;4)(p21;p16), der(5)t(5;5)(p15;?), der(11)t(3;11)(q11;q25) see additional details in methods). We generated allele-discriminating pyrosequencing assays to examine silencing of candidate genes flanking the integration sites (Fig. 3). The phase of the polymorphisms relative to the integration was not known, but the allelic expression change upon DOX induction of XIST is presented as a cis-linked loss of expression as

Table 1 Increased nucleolar association of chromosomes expressing transgenic XIST

\begin{tabular}{|c|c|c|c|c|c|c|}
\hline \multirow[t]{2}{*}{ Integration site } & $\%$ perinuclear & $\%$ perinuclear & $\%$ perinuclear & $\%$ perinucleolar & $\%$ perinucleolar & $\%$ perinucleolar \\
\hline & No DOX & DOX & CHANGE & No DOX & DOX & CHANGE \\
\hline $1 p$ & 27 & 34 & 7 & 10 & 36 & $16^{a}$ \\
\hline $3 q$ & 78 & 70 & -8 & 10 & 18 & 8 \\
\hline $4 q$ & 57 & 74 & $17^{\mathrm{b}}$ & 12 & 25 & $14^{\mathrm{a}}$ \\
\hline $7 p$ & 32 & 27 & -5 & 29 & 47 & $18^{\mathrm{a}}$ \\
\hline $7 q$ & 30 & 53 & $23^{a}$ & 28 & 39 & $11^{c}$ \\
\hline $8 p$ & 59 & 39 & $-21^{a}$ & 29 & 56 & $27^{\mathrm{a}}$ \\
\hline $12 q$ & 36 & 39 & 3 & 18 & 35 & $17^{\mathrm{a}}$ \\
\hline $15 q$ & 24 & 36 & $14^{c}$ & 46 & 53 & 7 \\
\hline Xq23 & 42 & 46 & 5 & 31 & 41 & 10 \\
\hline
\end{tabular}

The numbers shown are based on three independent experiments for the DOX results and one experiment for the No DOX results, with $\geq 50$ cells counted for each integration site in each experiment. Chi-squared test $\left({ }^{\mathrm{a}} P \leq 0.001 ;{ }^{\mathrm{b}} P \leq 0.01 ;{ }^{\mathrm{C}} P \leq 0.05\right)$ 


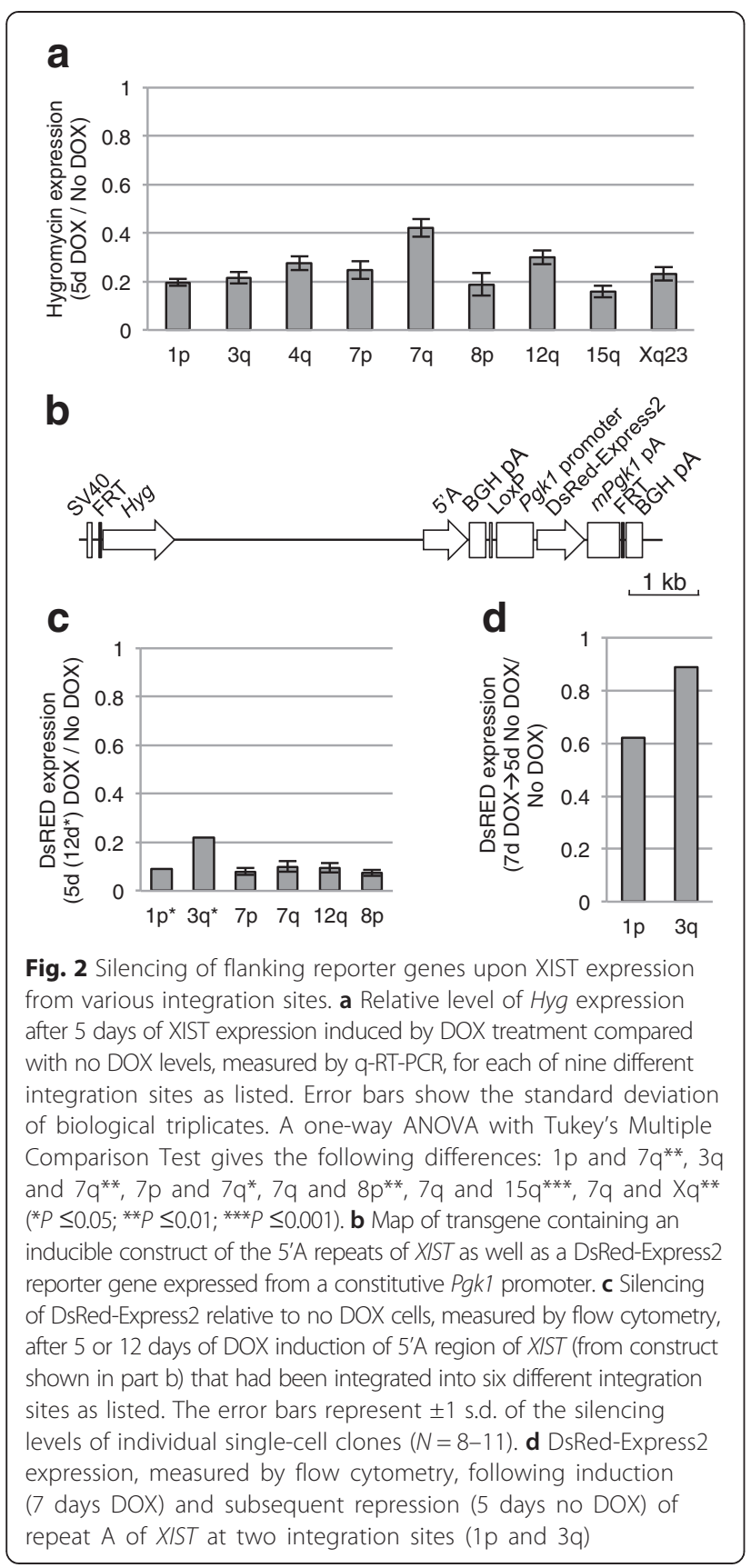

previously demonstrated for the $3 \mathrm{q}$ integration for which we were able to assign the allelic loss to the chromosome bearing the inducible XIST [40]. Individual pyrosequencing results for the locus closest to $5 \mathrm{Mb}$ from the integration site are shown in Fig. 3a, with the silencing percentages for all genes examined shown in Fig. $3 \mathrm{~b}$ as a function of distance from the integration site (all assays are shown in Additional file 3). While no significant changes were observed for the control integrations (clones with XIST integrated on different chromosomes), all XIST integration sites except 4q showed at least one gene with significant allelic silencing. There was much more variability between integration sites for endogenous gene silencing than was seen for the silencing of Hyg. The 8p-integrated XIST clone displayed the most silencing, with four out of the five genes tested showing 60-80 \% silencing. The 4q clone, in contrast, showed no significant silencing for any of the three genes tested. Two different integration sites on chromosome 7 showed quite different results, with only one of seven genes assayed showing over $20 \%$ silencing for the $7 \mathrm{p}$ integration site, while five of the seven genes showed over $20 \%$ silencing with XIST expressed from the $7 \mathrm{q}$ integration site. There was also discontinuous spread of silencing. For example, in the $1 p$ integration site clone, two genes located approximately $200 \mathrm{~kb}$ from the XIST transgene failed to silence (1 \% silencing), whereas the RHBDL2 gene located approximately $400 \mathrm{~kb}$ from the XIST transgene silenced by approximately $70 \%$. In addition to variation between the integration sites in the number of genes that were silenced, there were also significant differences in the extent of gene silencing between genes that showed silencing. More than half of the significant changes demonstrated less than $50 \%$ silencing of one allele, and a significant change as small as $6 \%$ for the ZNF710 gene on 15q was observed, indicating that XIST can cause a continuum of silencing.

To distinguish differences between clones that are attributable to the integration site rather than cloneto-clone variation, we analyzed clones that were independent integrations of the XIST cDNA into the same genomic location. Intriguingly, for the $12 \mathrm{q}$ integration site, we observed a significant correlation between the amount of silencing and the level of XIST expression (Fig. 3c). This correlation was observed for both cultures of the same single-cell clone and additional independent clones at the same integration site. Such a correlation was not observed for genes silenced by XIST expression from the $8 \mathrm{p}$ integration site using multiple cultures and two independent clones (Fig. 3d). Thus, while variation in the level of XIST expression occurred within a clone, the impact of this variation depended on the integration site, and with similar XIST expression a similar extent of silencing was observed between independent integrations into the same FRT site. A second clone from the Xq integration site showed very similar silencing, while a second clone from the $7 \mathrm{q}$ integration site showed lower XIST expression levels and failed to silence (Additional files 1 and 3), suggesting that the silencing ability of $7 q$ integrations, like $12 \mathrm{q}$ integrations, might be influenced by XIST expression levels. Since the clones at the $8 p$ integration site silenced across a range of XIST levels, it seemed possible that, unlike silencing of the reporter genes, the maintenance of silencing of endogenous genes might not be XIST-dependent, so we analyzed silencing of endogenous flanking genes after removal of DOX for the $8 p$ 
a
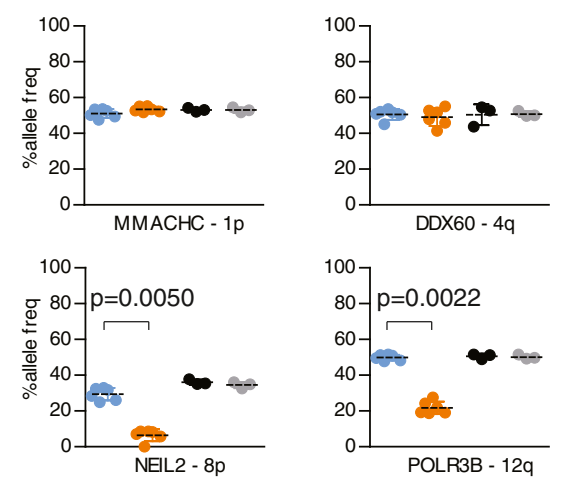

b

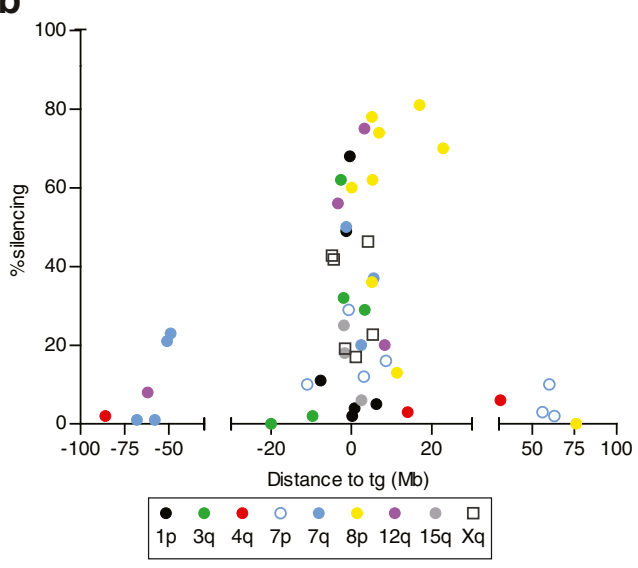

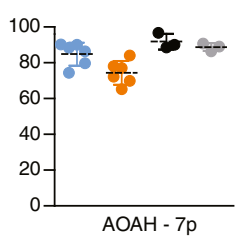

100

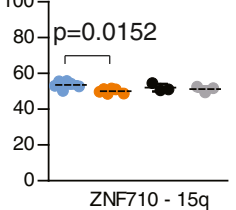

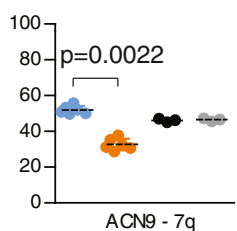

- Respective No DOX

- Respective DOX

- Control No DOX

- Control DOX

C



d

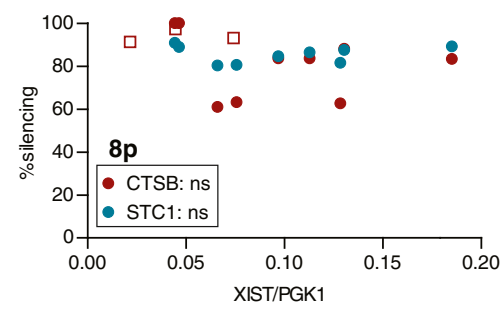

e
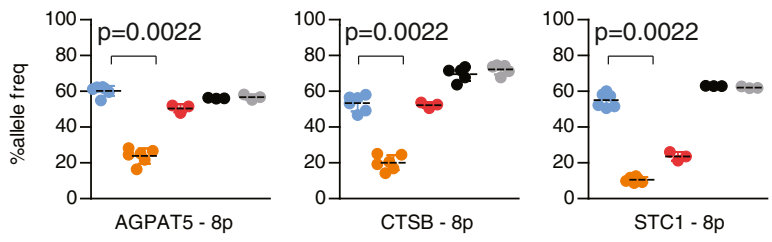

- No DOX - DOX - DOX $\rightarrow$ No DOX

- Control No DOX

- Control DOX

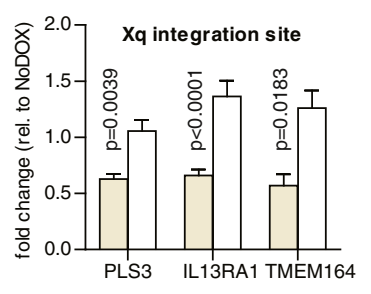

Fig. 3 Allelic silencing of flanking endogenous genes upon XIST induction. a Allele-discriminating RT-PCR pyrosequencing assay for genes closest to $5 \mathrm{Mb}$ of integration site for each integration site, comparing triplicate cDNAs from untreated cells (No DOX) and following 5-day DOX induction of XIST in duplicate pyrosequencing reactions. CDNA from a different integration was also assessed (additional assays are shown in Additional file 3). $P$ values of significantly silenced genes are listed. $\mathbf{b}$ Summary of the silencing observed for individual genes for each of the nine integration sites (color-coded as shown in the legend); plotted by distance from the integration site on the chromosome (from short to long arm). The allelic change is shown as percent silencing, which was calculated as: (allele frequency No DOX - allele frequency $5 \mathrm{~d}$ DOX)/ allele frequency No DOX $\times 100$ ) for the pyrosequencing assays. For the Xq integration the silencing was determined by q-RT-PCR since the chromosome is hemizygous. Phase was determined for the $3 q$ integration but for all integrations the allelic change is shown as silencing. Integrations on other chromosomes showed no silencing upon DOX induction. c Correlation between extent of silencing and level of XIST RNA. Five different clones (symbols) and cultures show variation in the level of XIST RNA after DOX induction (as measured by qRT-PCR for XIST relative to PGK1), and for 12q this correlates well with the extent of silencing of two genes assayed by allelic pyrosequencing after RT-PCR (OAS3, $P<0.0001 ;$ POLR3B, $P=0.0004$ ). A similar analysis (d) for the chromosome $8 p$ integration site showed a similar variation in XIST levels, but no correlation with extent of silencing of two loci on 8p. e Removal of XIST expression after 5-day DOX induction resulted in substantial reactivation of endogenous genes in the $8 p$ and $\mathrm{Xq}$ integration sites 
and $\mathrm{Xq}$ integration sites. All genes examined showed partial to complete reactivation (Fig. 3e) 5 days after induction of XIST had ceased, indicating that the silencing observed requires ongoing XIST expression.

\section{RNA-seq confirms differences in silencing capacity of XIST at different integrations}

The significant differences between the integration sites in their capacity to silence endogenous genes suggested an important impact of the genomic context of the integration; however, it was possible that by chance the genes chosen to be tested were non-random in their ability to silence. Therefore, we chose to examine three clones with XIST at different integration sites using RNA-seq to generate a detailed view of any variability in silencing capacity. We chose to examine the $8 p$ and $12 q$ integration sites, which had shown the most silencing, but different sensitivities to XIST levels, as well as the $1 p$ integration site, which had shown limited silencing.

As our candidate genes examined by pyrosequencing had shown significant reductions in gene expression that ranged from as little as $6 \%$ to approximately $80 \%$ silencing of one allele, we did not expect complete silencing of one allele that would reduce expression levels overall by $50 \%$. We chose a stringent threshold of 30-60\% total reduction to classify genes as silencing, at which level a significantly greater proportion of genes were observed within $30 \mathrm{Mb}$ of the $8 \mathrm{p}$ and $12 \mathrm{q}$ integration sites compared to the genome (Chi-square test, $P<0.0001$ ). Consistent with our candidate gene analysis, the increase in proportion of genes in this range was not significant for the $1 \mathrm{p}$ integration site. Examination of expression changes as an allelic change is more sensitive to the partial reduction of expression of one allele, and should also still detect changes in allelic expression when the total expression level of the gene is regulated in trans although the number of genes that can be examined is reduced by the requirement for an expressed polymorphism. We examined the allelic change in expression on chromosomes 1,8 , and 12 (Fig. 4), and again a significantly higher proportion of genes showing an allelic change of greater than $30 \%$ was seen for genes flanking the $8 \mathrm{p}$ integration site. A Chi-square permutation test demonstrated that the number of genes silenced on $8 \mathrm{p}$ following XIST induction decreased with increasing distance $(P=0.008)$. The biological duplicate for RNA-seq was highly concordant for the percent allelic gene silencing for 8p (Spearman $r=0.5806 ; P<0.0001$ for genes with FPKM $\geq 5$ ). The proportion of genes showing allelic silencing for genes flanking the $1 \mathrm{p}$ integration site was significant $(P=0.0269)$; however no significant change was seen for the $12 q$ integration site. We show only the distal end of chromosome 1 as the HT1080 cells carry a translocation of one chromosome 1 to chromosome 4

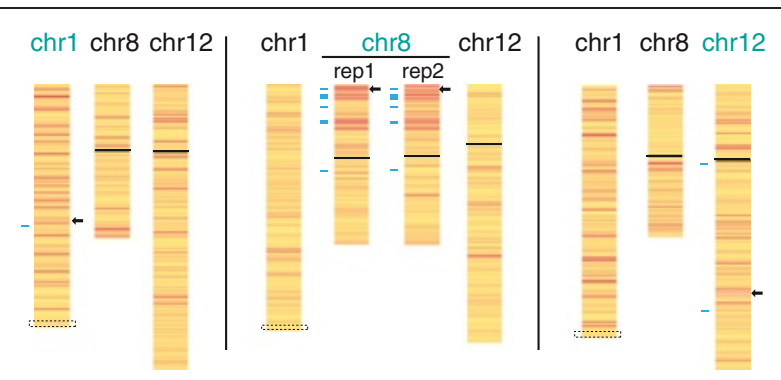

Fig. 4 Silencing of endogenous genes upon ectopic XIST expression. Allelic inactivation of chromosomes 1, 8, and 12 is shown as a heat map following DOX induction of XIST in cells containing integrations into the chromosome listed in green. Colors denote the allelic expression change as assessed by RNA-seq (red = downregulation; yellow $=$ no change) for those genes with allelic reads with FPKM $\geq 5$. The integration site is marked as a black arrow; confirmatory pyrosequencing assays are indicated by blue lines, and the centromere is shown as a black line. For chromosome 1, the chromosome is truncated at the site of a translocation as it is unknown whether the integration is on the translocated chromosome (boxed region)

(approximately $55 \mathrm{Mb}$ distal to the integration site). We validated several of the observed changes by pyrosequencing or q-PCR, and the assays are included in Additional file 3, and highlighted on Fig. 4. Percent allelic silencing was highly concordant between pyrosequencing and RNA-seq $(r=0.9341 ; P<0.0001)$. Of the 17 genes showing at least $50 \%$ allelic silencing in both 8p RNA-seq replicates, two genes (DLC1 and STC1) did not show a decrease in total expression, suggesting that auto-regulation could be another source of discrepancy between total read and allelic read changes. The high rate of validation of the observed changes by pyrosequencing of biological triplicates substantiated that silencing spread to endogenous genes. Intriguingly, on chromosome 8 there appeared to be domains of silencing separated by areas that were more resistant to the action of XIST. In order to explore what features might lead to differential susceptibility to silencing we performed chromatin immunoprecipitation followed by sequencing (ChIP-seq) for induced and uninduced cells with XIST integrated at 8p.

\section{Chromatin features of regions silenced by XIST in somatic cells}

ChIP-seq for both the archetypical facultative heterochromatic mark H3K27me3 and the active mark H3K27ac showed significant, but opposing changes on chromosome 8 upon DOX induction of XIST from the $8 p$ integration site, and these changes were even more dramatic on the short arm where XIST is integrated (Fig. 5a, b). Surprisingly, DOX treatment significantly increased H3K27ac across the genome, with most chromosomes showing an increase, suggesting a widespread impact of the antibiotic (Fig. 5b). This unexpected change in chromatin accessibility did not extend to any significant 


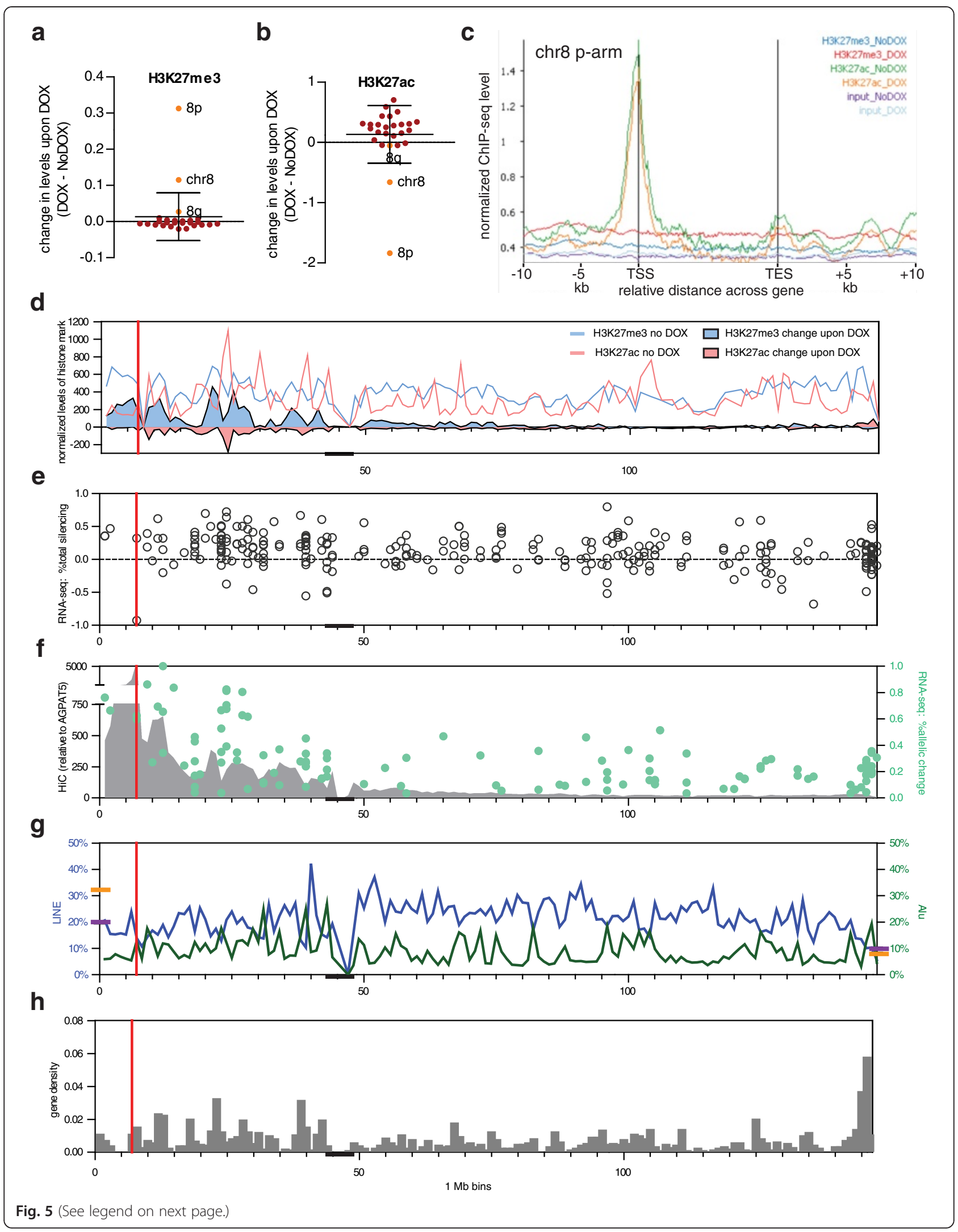


(See figure on previous page.)

Fig. 5 Correlation of genomic neighborhood and XIST-induced silencing on chromosome 8. a ChIP-seq changes observed for H3K27me3 on each chromosome, with chromosome 8 also subdivided into $8 p$ (arm with integration) and 8q. H3K27me3 showed significant $\left(P=3.3 e^{-243}\right.$, paired $t$-test) changes for chromosome 8 with the genome showing no significant change. b ChIP-seq changes observed for H3K27ac on each chromosome, with chromosome 8 also subdivided into $8 p$ (arm with integration) and $8 q$. The chromosome 8 decrease $\left(P=2.2 e^{-40}\right)$ as well as the genome-wide increase $\left(P=2.0 \mathrm{e}^{-205}\right.$ ) were both highly significant. c Average H3K27ac and H3K27me3 normalized across genes on 8p. Normalized ChIP-seq level are shown across genes and for the $10 \mathrm{~kb}$ upstream and downstream of genes before XIST expression (NoDOX) and after XIST expression (DOX) as well as for input, color-coded as outlined. d Total H3K27me3 (blue line) and H3K27ac (pink line) in No DOX overlaid with change in H3K27me3 (blue), H3K27ac (pink) along chromosome 8 after 5 days of DOX induction of XIST. e Change in total reads for genes with average $\geq 5$ FPKM shown as percent silencing. $\mathbf{f}$ Density of nuclear contacts with the $8 p$ integration site (AGPAT5) as identified in IMR90 fibroblast cells by HiC [45] in $1 \mathrm{Mb}$ bins (grey shading). Allelic silencing of genes is shown superimposed as green dots. $\mathbf{g}$ Density of LINE (blue line) and ALU (green line) repetitive elements per $1 \mathrm{Mb}$ bin. Shown on the axes are the average genome (purple) and X-chromosomal (orange) densities of the elements. $\mathbf{h}$ Gene density in $1 \mathrm{Mb}$ bins along the chromosome

change in $\mathrm{H} 3 \mathrm{~K} 27 \mathrm{me} 3$ across the genome, and the anticipated enrichments in the imprinted regions of KCNQ1 and IGF2R were observed before and after DOX (Additional file 4). When examined across all genes on chromosome $8 \mathrm{p}$, the loss of acetylation was most notable at the promoter, the site of most pre-existing acetylation, but loss was seen throughout the upstream and gene bodies (Fig. 5c). The genes from $8 \mathrm{q}$ showed changes similar to that of other autosomes, with an increase only detected at the promoter (Additional file 5). The gain of H3K $27 \mathrm{me} 3$ on chromosome $8 \mathrm{p}$ was observed across both genic and intergenic regions (Fig. 5c), with no change observed for genes on $8 \mathrm{q}$ or autosomes (Additional file 5). In order to correlate the change in H3K27me3 and H3K27ac with the silencing observed, as well as other features of the chromosome, we plotted the initial levels (without DOX) as well as the total change in both marks along the chromosome (Fig. 5d). While the loss of H3K27ac could be anticipated to occur from locations where there was acetylation initially, the recruitment of H3K27me3 upon XIST induction also mirrored the pre-existing levels remarkably well (Spearman $r=0.8671 ; P<0.0001$ for $8 p$ ). In Fig. 5e we show the average silencing determined from total reads, while in Fig. $5 \mathrm{f}$ we show allelic gene silencing. We assessed allelic changes in H3K27ac as well, and the limited informative genes showed a significant correlation between allelic silencing and loss of acetylation (correlation for 29 genes with data for both, $r=0.5904 ; P=0.0007$ ). Mouse Xist localizes to sequences that are in contact with the integration site as determined by chromatin conformation capture $[43,44]$, and therefore we extracted the $\mathrm{Hi}-\mathrm{C}$ contacts anchored at the $1 \mathrm{Mb}$ domain containing the $8 \mathrm{p}$ integration site from the published Hi-C data [45] (Fig. 5f). There are more contacts, as well as stronger silencing, closer to the integration site, confounding the ability to examine correlations. Interestingly, the $\mathrm{Hi}-\mathrm{C}$ contacts correlated with the pre-existing (No DOX) H3K27me3 levels along the chromosome 8 short arm (Spearman $r=0.4996$; $P=0.0006)$, although we did not identify an association of allelic silencing with domains designated as closed nor open [46] (Fisher's exact test). Allelic silencing correlated with both the gain of H3K27me3 (Spearman $r=$ 0.4599; $P=0.0003$ ) and loss of acetylation (Spearman $r=$ $-0.4557, P=0.0004)$. Given the proposed role for repetitive elements in the XCI process [47-49], and the tendency for mouse Xist to first localize to gene-rich regions during early expression in embryonic stem cells [43, 44] we show the LINE and ALU distribution (Fig. 5f) and the gene density (Fig. 5g) along chromosome 8; however, neither feature showed a significant correlation with silencing, although as would be expected, gene density is a major contributor to total levels of H3K27 acetylation. To further explore the relationship of the ability to modify chromatin and the silencing ability of the different chromosomal integration sites we performed immunofluorescence in conjunction with FISH for XIST.

\section{Recruitment of heterochromatic modifications to the site of XIST localization}

In addition to $\mathrm{H} 3 \mathrm{~K} 27 \mathrm{me} 3$, the $\mathrm{Xi}$ is known to be enriched for additional histone marks and proteins associated with repressed chromatin; however, the hierarchy of recruitment of these heterochromatic features by XIST has not been clarified. Therefore, we examined the co-localization of these features with XIST expressed from various integration sites (Table 2). The extent of co-localization can vary with the cell cycle, and therefore we categorize the co-localization as positive (consistently observed in greater than $25 \%$ of XIST-positive cells), negative (consistently observed in less than $10 \%$ of cells) or $+/-$ (in between $10 \%$ and $25 \%$ co-localization, or variable between replicates). No recruitment of H3K9me3 was observed at the four sites examined, while H2AK119u1 was observed at all four examined integration sites. The integration onto Xq showed recruitment of all marks (with the exception of H3K9me3) and is shown in Fig. 6. Recruitment was still less than was seen for a female cell line - in IMR90 we routinely detect over $80 \%$ co-localization. H3K27me3 was not recruited to the $1 \mathrm{p}$ or $3 \mathrm{q}$ integration sites, while SMCHD1 was not enriched at 1p, 3q, and also 
Table 2 Co-localization of histone modifications with induced XIST RNA signal

\begin{tabular}{|c|c|c|c|c|c|c|}
\hline Clone & H3K27me3 & H4K20me1 & macroH2A & SMCHD1 & H2AK119u1 & H3K9me3 \\
\hline$\overline{1 p}$ & - & $+/-$ & $-^{a}$ & - & + & - \\
\hline $3 q$ & - & & $+^{a}$ & $--^{\mathrm{a}}$ & & \\
\hline $4 q$ & $+/-$ & - & $+/-$ & $-^{a}$ & & \\
\hline $7 p$ & $+/-$ & $+/-$ & + & $+/-^{a}$ & & \\
\hline $7 q$ & $+/-^{b}$ & $-{ }^{\mathrm{b}}$ & $-^{a}$ & $+/-$ & & \\
\hline $8 p$ & $+{ }^{\mathrm{b}}$ & $+/-^{\mathrm{b}}$ & $+{ }^{a}$ & $+^{\mathrm{a}}$ & + & - \\
\hline $12 q$ & $+/-^{\mathrm{b}}$ & $-^{a}$ & + & $+/-$ & + & - \\
\hline $15 q$ & $+/-$ & $+/-$ & $+/-$ & $+/-$ & & \\
\hline$X q$ & + & $+t^{b}$ & $t^{a}$ & + & + & - \\
\hline
\end{tabular}

${ }^{a}$ In situ hybridization performed at least in duplicate

${ }^{b}$ In situ hybridization performed on two independent clones

Co-localization of the histone modification with the XIST signal was counted in at least 30 cells. No co-localization (-) was $<10 \%$ co-localization; $+/-$ was between $10 \%$ and $25 \%$ co-localization and + is assigned to integration sites with over $25 \%$ co-localization

$4 \mathrm{q}$ integration site clones. The integration clone at $3 \mathrm{q}$ did however recruit macroH2A, which was not seen to be enriched at the $1 p$ or $7 q$ integration clones. Overall the integration at $1 \mathrm{p}$ showed the least enrichment of marks with only variable recruitment of $\mathrm{H} 4 \mathrm{~K} 20 \mathrm{me} 1$ in addition to H2AK119u1. To further examine dependence on integration site, independent clones integrated into $7 \mathrm{q}, \mathrm{Xq}$, and $8 \mathrm{p}$ were compared by IF-FISH for H3K $27 \mathrm{me} 3$ and/or H4K20me1. Of the six side-by-side comparisons of independent clones at the same integration site, enrichment of marks were very comparable showing an average of $6 \%$ difference in enrichment and all falling within the same category (except for 8p with H4K20me1 which spanned the $+/-$ and + ).

The only mark examined that was not detected at any site was the H3K9me3 mark, which is often associated with constitutive heterochromatin. H2AK119u1 was seen at all of the four integrations examined, suggesting it is less dependent on local chromatin structure than the other marks which were heavily dependent upon

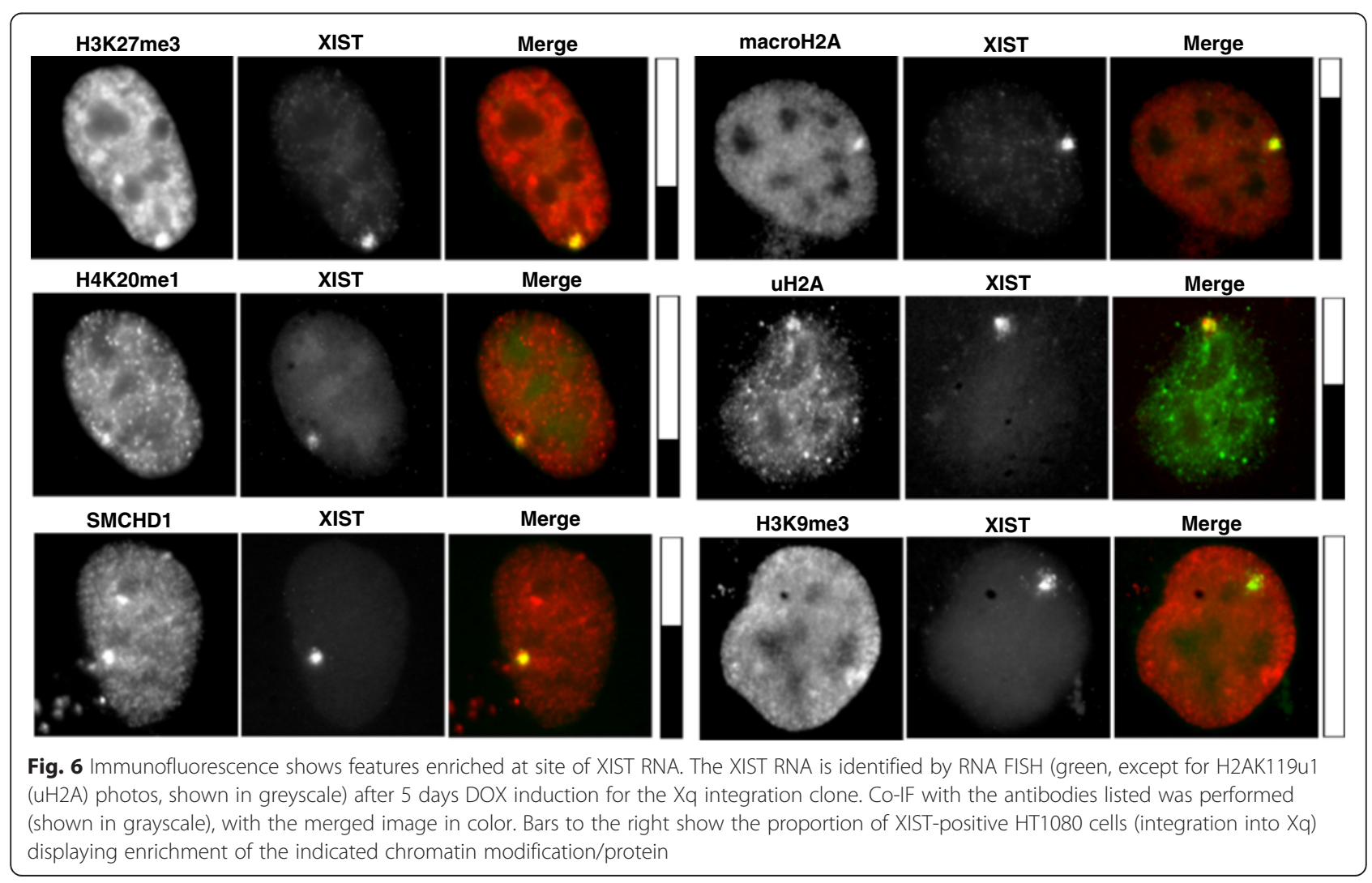


the integration site and surprisingly independent of each other. The X chromosome showed the best ability to recruit all features examined, with the next best integration site also being the one that showed the greatest gene silencing $(8 \mathrm{p})$.

\section{Discussion}

We have examined the impact of genomic location of XIST on its ability to alter nuclear ultrastructure, chromatin state, and gene expression in a somatic cell line. We observe considerable heterogeneity in the extent of silencing and the recruitment of chromatin marks, depending upon the integration site, providing us with an opportunity to dissect the interactions between features in a system in which complete recruitment and silencing does not occur. The ability of XIST to localize to all integration sites supports that there is limited sequence specificity for the RNA with the chromosome, in agreement with the spread of inactivation reported in $\mathrm{X} /$ autosome translocations, and various mouse Xist transgenes (reviewed in $[36,50]$ ). The lack of sequence specificity is also in line with the recently described proximity-transfer model which suggests that Xist first associates with sequences in physical proximity to the Xist locus and then transfers to gene-rich regions which are topologically associated with the integration site [43].

Spread of silencing to autosomes in these HT1080 cells is not seen to the extent that is observed for X/ autosome translocations $[48,49]$ or an autosomal XIST transgene in iPS cells [39], suggesting that bypassing differentiation reduces the ability to inactivate a chromosome. While early mouse studies suggested the presence of a limited developmental window during which silencing could be induced [12] we see XIST-dependent silencing of endogenous genes in these HT1080 fibrosarcoma cells up to almost $50 \mathrm{Mb}$ from the integration site, although endogenous gene silencing is most prominent closer to the integration site. It is possible that the cancerous origin of these cells has reactivated critical developmental gatekeepers, as specific mouse cancers have been shown to allow Xist-induced silencing $[17,25]$. The ability of XIST to induce gene silencing in somatic cells has important implications for cancer cells where rearrangements or reactivation of XIST may bring previously active genes under the influence of XIST.

We observe a Cot-1 hole and increased perinucleolar association at all integration sites, consistent with previous reports that XIST/Xist expression from autosomes increases perinucleolar association $[10,21]$. In our assessment of multiple XIST integrations, we observed heterogeneity in the extent of perinucleolar association, and the correlation with silencing was limited. The three integration sites for which the increase in perinucleolar association was not significant $(15 \mathrm{q}, 3 \mathrm{q}$, and $\mathrm{Xq}$ ) were all G-dark integration sites; however, the $7 p$ integration was also in a G-dark band yet demonstrated a significant increase in perinucleolar localization, suggesting an incomplete association between perinucleolar association and G-dark integrations with lower XIST levels (Fig. 7).

The other feature that we observed at all integrations examined was H2AK119u1, which is established by the PRC1 complex. We observed H2AK119u1 in the absence of SMCHD1, macroH2A, and H3K27me3 in the $1 \mathrm{p}$ integration cells, suggesting that XIST may be able to directly recruit the PRC1 complex, in agreement with the PRC2-independent PRC1 recruitment previously suggested in mice [27]. As the $1 \mathrm{p}$ integration site demonstrated limited silencing in the presence of H2AK119u1 recruitment, we conclude that $\mathrm{H} 2 \mathrm{AK} 119 \mathrm{u} 1$ is insufficient for the spread of gene silencing. No integration site examined showed recruitment of H3K9me3, although the antibody clearly hybridized to the native $\mathrm{Xi}$ in female cells. Therefore it appears that facultative heterochromatic marks can be recruited by XIST in these cells, but the establishment of a 'locked-in' silent state required additional layers of developmentally-regulated chromatin condensation. Without such locks on silencing, the continued silencing of both reporter genes and endogenous genes was XIST-dependent, undergoing reactivation upon removal of XIST induction.

Interestingly, no single factor was seen to associate with more robust silencing (Fig. 7), demonstrating considerable redundancy in XIST-inducible silencing pathways. Consistent with previous studies in mice, silencing of endogenous genes in these human cells was observed in the absence of recruitment of macroH2A (7p) [30], SMCHD1 (3q) [51], or H3K27me3 (12q) [27]. MacroH2A has been reported to be recruited by induction of mouse Xist expression [13], and is also lost upon deletion of Xist [14]; yet intriguingly we observe macroH2A recruitment to be site-dependent, rather than solely XIST-dependent. We observed that macroH2A is not required for XISTinduced silencing, which is consistent with the ability of mice that are knocked-out for both macroH2A1 and macroH2A2 to continue to undergo XCI [30]. The recruitment of SMCHD1 and macroH2A seems to be independent of each other as, for example, the $3 \mathrm{q}$ integration site recruits macroH2A but not SMCHD1, while the $7 q$ integration shows some SMCHD1 recruitment, but no macroH2A recruitment. There was a positive correlation between the amount of SMCHD1 recruitment and the final perinucleolar localization (Spearman $r=0.8$; $P=0.01$ ), with the proportion of cells positive for SMCHD1 always equal or lower to the percent seen to be perinucleolar. Thus perinucleolar localization may be required but not sufficient to recruit SMCHD1. 


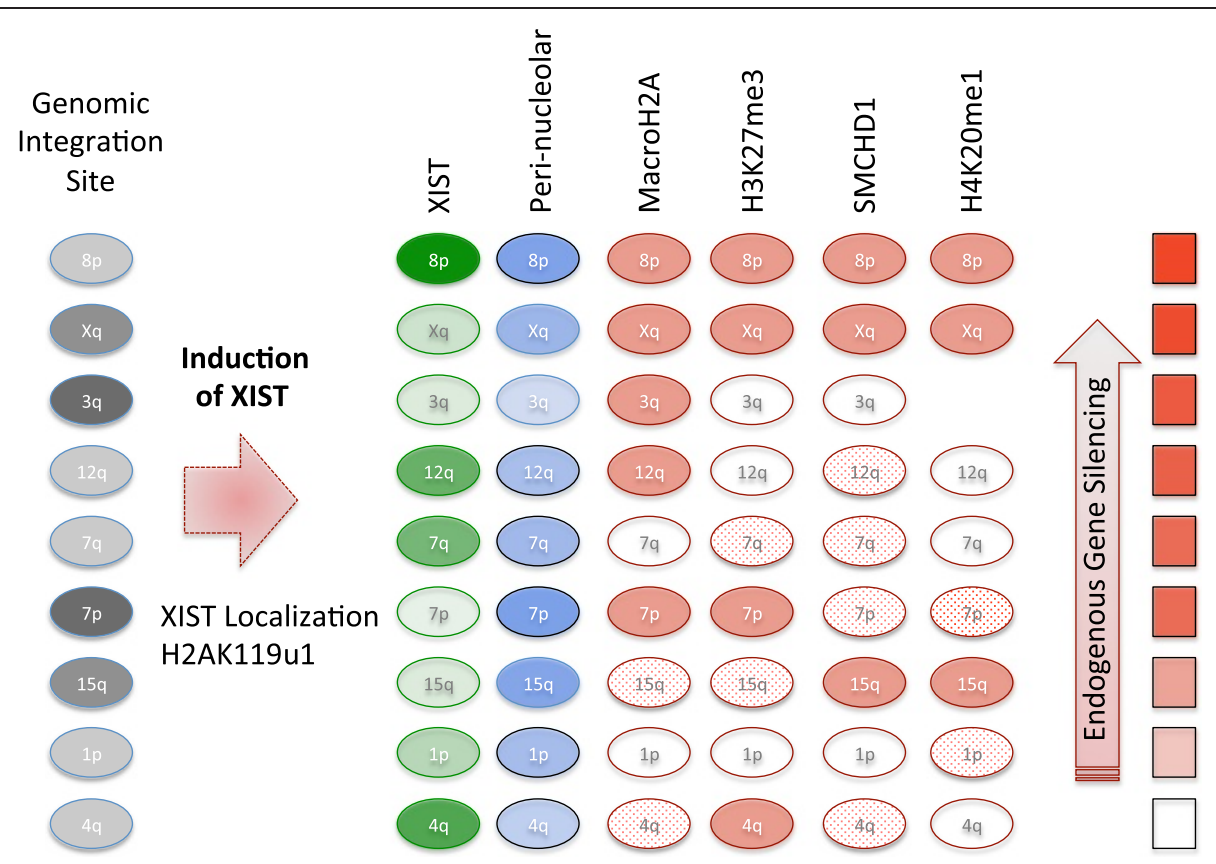

Fig. 7 Schematic of features examined at the site of XIST RNA induction. Nine different integration sites of XIST were examined, and these were in both G-light (pale gray) and G-dark genomic locations. Upon DOX induction XIST was expressed (intensity of green oval reflects average amount of XIST expression) and increased perinucleolar localization was observed (blue oval intensity reflects increase, with significant changes encircled in black). H2AK119u1 was enriched at all four integration sites examined. The enrichment of chromatin marks or proteins that were variably recruited (see Table 2) is shown as solid (enrichment $>25 \%$ ), dotted (enrichment between $10 \%$ and $25 \%$ ) or unfilled (enrichment $\leq 10 \%$ ). The integration sites are ordered by ranking of gene silencing observed by pyrosequencing (fill of red rectangle reflecting proportion of silenced genes, see Additional file 3)

The strong ability of the $\mathrm{X}$ integration to recruit all features confounded the ability to detect correlations; however, it appears that the majority of the features are independently recruited to the chromosome that expresses XIST. For several of the integration sites two or more independent clones with similar XIST levels behaved similarly supporting that the variation we observe is due to the integration site. In addition, the $4 \mathrm{q}$ and $12 \mathrm{q}$ clones were derived from a different subclone of HT1080 from the other autosomal integration sites; however, they did not appear to be more similar to each other, arguing against the variation arising during subclone generation.

The differential recruitment of chromatin marks depending on integration site suggests that multiple silencing pathways not only work in parallel to promote silencing, but that their recruitment is favored by different underlying DNA sequences, consistent with previous studies using genes that escape from inactivation to identify 12 features of the DNA sequence of the X chromosome that may be involved in the spread or maintenance of XCI [52].

\section{Conclusions}

Overall, we saw variability in the recruitment of chromatin marks between the integration sites, highlighting the importance of the integration site in modulating XIST function. Localization of XIST with a concomitant depletion of Cot-1 RNA, recruitment of H2AK119u1, and a shift to perinucleolar location was seen at all integrations, and thus reflect features established by XIST independent of the local chromatin environment. In contrast, recruitment of SMCHD1, macroH2A, H3K27me3, and H4K20me1 appeared to be strongly influenced by the site of XIST expression. As some silencing was observed at all integrations, this study demonstrates that silencing in human somatic cells can occur in the absence of macroH2A, SMCHD1, and H3K27me3/H4K20me1 recruitment, underscoring the independent but cooperative nature of the $\mathrm{X}$-chromosome inactivation process. The $\mathrm{X}$ chromosome demonstrated the most consistent ability to recruit the heterochromatic marks of XCI, consistent with an evolutionary accumulation of DNA features enabling the recruitment of heterochromatic marks to the $\mathrm{X}$ chromosome.

\section{Methods}

Generation and culture of cell lines and identification of the transgene integration site

HT1080 HH1 cells were transfected with pcDNA6/TR, and two subclones (HT1080HH1-2-3 or 2-12) expressing 
high RNA levels of the Tet-repressor were subsequently transfected with pFRT/lacZeo (Life Technologies) at low concentrations. The full-length inducible XIST cDNA construct [20] was co-transfected with the pOG44 plasmid expressing Flp recombinase for site-specific recombination into the FRT site followed by Hygromycin selection and confirmation of loss of Zeomycin resistance. Cells were grown at $37{ }^{\circ} \mathrm{C}$ with $5 \% \mathrm{CO}_{2}$ in DMEM supplemented with penicillin/streptomycin, non-essential amino acids, and $10 \% \mathrm{~V} / \mathrm{V}$ fetal bovine serum. XIST expression was induced with the addition of $1 \mu \mathrm{g} / \mathrm{mL}$ doxycycline to the culture medium.

Southern blotting identified eight FRT integrations that appeared single copy. Inverse PCR utilizing primers complementary to a sequence within the integrated pFRT plasmid was used to identify the precise integration site of the transgenes in the HT1080 cell lines. The ends of linearized plasmids are subject to exonuclease activity, thus the actual integrated transgene often lacks several hundred of base pairs on each end which was first identified by a series of PCR assays prior to restriction endonuclease digestion with a frequently-cutting restriction endonucleases identified to cut in the remaining plasmid, followed by ligation with T4 DNA ligase (Invitrogen) to create circular DNA molecules. The captured genomic DNA was amplified by nested PCR with primers facing outward from the plasmid fragment for sequencing and the genomic location was identified using the BLAT algorithm [53]. No X-linked integration was identified, so the F55 HT1080 clone from Yan and Boyd was used [41].

While cancer derived, the HT1080 cells remain diploid with four structural rearrangements detected by spectral karyotyping (46,XY,del (1)(p21), i(3)(p10), $\mathrm{i}(3)(\mathrm{q} 10), \quad \operatorname{der}(4) \mathrm{t}(1 ; 4)(\mathrm{p} 21 ; \mathrm{p} 16), \quad \operatorname{der}(5) \mathrm{t}(5 ; 5)(\mathrm{p} 15 ; ?)$, $\operatorname{der}(11) t(3 ; 11)(\mathrm{q} 11 ; \mathrm{q} 25)$. Using our allelic pyrosequencing assays we observed instability of chromosome 3 in two of nine clones (see also [40]) and homozygosity for assays on $4 \mathrm{q}$ in one clone, while assays on chromosomes $1,7,8$, and 15 remained diploid in the clones tested, suggesting that the unbalanced rearranged chromosomes were the least stable in these cells. We analyzed two independent clones at the $\mathrm{Xq}, 7 \mathrm{q}$, and $8 \mathrm{p}$ integration site and five independent clones of the $8 \mathrm{p}$ integration site. These clones were individual single-cell clones following Flp-mediated recombination into the FRT site. Each clone was selected for Hyg-resistance and assessed for loss of Zeomycin sensitivity.

As fluorescent reporters allow for efficient screening, we created a plasmid that carries both the inducible repeat A and a DsRed-Express2, driven by the mouse Pgk1 promoter (Fig. 2b). To test whether the ability of repeat A to silence the reporter depends on the genomic integration site, we inserted the repeat A - DsRed-Express2 transgene into six of the HT1080 cell lines with a known chromosomal location of the FRT integration site for assessment of silencing by flow cytometry as previously performed [40].

\section{RNA FISH and immunofluorescence}

Cells were grown on glass coverslips. Upon removal from cell culture the coverslips were first rinsed in icecold CSK buffer $(0.3 \mathrm{M}$ sucrose, $100 \mu \mathrm{M} \mathrm{NaCl}, 10 \mu \mathrm{M}$ PIPES, $3 \mu \mathrm{M} \mathrm{MgCl}_{2}$ ), then permeabilized with $0.5 \%$ Triton-X 100 in CSK for $8 \mathrm{~min}$ on ice and then fixed in $4 \%$ paraformaldehyde for $8 \mathrm{~min}$ at room temperature. Coverslips were stored at $4{ }^{\circ} \mathrm{C}$ in $70 \%$ ethanol. Just prior to RNA FISH, the coverslips were immersed in $100 \%$ ethanol for $5 \mathrm{~min}$ and left to air dry. FISH was performed with two probes: an XIST probe and a Cot-1 (Invitrogen) probe. Both probes had been directly fluorescently labeled using the Nick Translation Reagent Kit (Abbott Molecular, Inc.) with Spectrum red-UTP (Vysis) for Cot-1 DNA probes and Spectrum green-UTP (Vysis) for XIST probes. Approximately $150 \mathrm{ng}$ of each probe was mixed together along with $20 \mu \mathrm{g}$ salmon testes DNA then air dried in a speed vacuum, resuspended in $10 \mu \mathrm{L}$ deionized formamide, denatured at $80{ }^{\circ} \mathrm{C}$ for $10 \mathrm{~min}$, and then mixed with $10 \mu \mathrm{L}$ hybridization buffer (20 mg/mL BSA, 4XSSC, $20 \%$ ). This was pipetted onto a small square of Parafilm and the coverslip was placed on top of the probe mixture. Another piece of Parafilm was then placed on top and the edges were sealed to prevent the drying out of the coverslip. Hybridization took place overnight in a humidified chamber at $37{ }^{\circ} \mathrm{C}$. The next day the coverslips were rinsed as follows: $20 \mathrm{~min}$ in $50 \%$ formamide $/ 50 \%$ XXSC at $37{ }^{\circ} \mathrm{C}$, $20 \mathrm{~min}$ in $2 \mathrm{X} \mathrm{SSC}$ at $37^{\circ} \mathrm{C}$, and $20 \mathrm{~min}$ in $1 \mathrm{X} \mathrm{SSC}$ at room temperature. Coverslips were then stained with DAPI and mounted onto microscope slides with Vectashield (Vector Laboratories). Cells were observed on a Leica inverted microscope (DMI 6000B) at 100X magnification and images were obtained using a Retiga 4000R (Q-Imaging) camera with Openlab software (PerkinElmer). Images were processed using Adobe Photoshop CS4 to reduce background and correct for variation in FISH efficiency between different images. A one-way ANOVA test in GraphPad was used to determine significantly different signal sizes. Line scans were generated using Image J software (NIH) by drawing a line through the area of interest and plotting the RGB intensities across the line.

To determine the nuclear location, XIST signals were scored visually in Photoshop as being either 'perinuclear only' (for example, adjacent to and in contact with the nuclear periphery), 'perinucleolar only' (that is, adjacent to and in contact with a Cot-1 negative nucleolus), 'both' (that is, adjacent to and in contact with both the nuclear 
periphery and a Cot-1 negative nucleolus), or 'neither'. XIST signals scored as 'both' are included in the 'perinuclear'/'perinucleolar' percentages in Table 1. For the ' $5 \mathrm{~d}$ DOX' counts, results for each integration site are the average of three independent experiments performed on different coverslips, by at least two independent observers, with a minimum of 50 cells counted each time. The 'No DOX' counts were done once with a minimum of 60 cells counted per integration site.

For combined RNA FISH and immunofluorescence, coverslips, which had been stored at $4{ }^{\circ} \mathrm{C}$ in $70 \%$ ethanol, were first rinsed in PBS then placed onto a small amount of PBT (PBS with $1 \%$ BSA and $0.1 \%$ Tween 20) containing 0.4 U/ $/ \mu \mathrm{L}$ Ribolock RNase inhibitor. Coverslips were sealed between two layers of Parafilm and left in the blocking buffer at room temperature for $20 \mathrm{~min}$, then transferred from blocking buffer to PBT containing 1:100 primary antibody and $0.4 \mathrm{U} / \mu \mathrm{L}$ Ribolock, sealed between two layers of Parafilm and left at room temperature for 4-6 h. Coverslips were then washed three times, for $5 \mathrm{~min}$ each time, at room temperature in PBS containing $0.1 \%$ Tween 20, then put onto a small amount of PBT containing 1:250 fluorescently labeled secondary antibody and $0.4 \mathrm{U} / \mu \mathrm{L}$ Ribolock, sealed between two layers of Parafilm and left at room temperature in the dark for $45 \mathrm{~min}$. Coverslips were then washed three times, for $5 \mathrm{~min}$ each time, at room temperature in the dark in PBS containing $0.1 \%$ Tween 20. Coverslips were then fixed in $4 \%$ PFA in PBS for $10 \mathrm{~min}$ at room temperature in the dark, and washed for $5 \mathrm{~min}$ in PBS before continuing on to RNA FISH, making sure that the coverslips remained in the dark throughout the RNA FISH procedure. Antibodies used in immunofluorescence include: anti-H3K27me3 (07-449 from Millipore); anti-macroH2A (07-219 from Millipore); anti-SMCHD1 (ab31865 from Abcam); anti-H2AK119u1 (05-678 from Millipore); anti-H3K9me3 (07-442 from Millipore); anti-H4K20me1 (07-440 from Millipore).

\section{RNA isolation, reverse transcription Q-PCR and allelic discrimination by pyrosequencing}

RNA was isolated from cell pellets stored at $-70{ }^{\circ} \mathrm{C}$ using TRIZOL (Invitrogen) according to the manufacturer's instructions and then treated with DNase1. cDNA was generated in the range of $0.5-2.5 \mu \mathrm{g}$ RNA using M-MLV reverse transcriptase for $\mathrm{qPCR}$ on a StepOnePlusTM Real-Time PCR System (Applied Biosystems, Darmstadt, Germany), using Maxima Hot Start Taq (Thermo Scientific) and EvaGreen dye (Biotium). The following conditions were used: $95^{\circ}$ for $5 \mathrm{~min}$, followed by 40 cycles of $\left(95^{\circ}\right.$ for $15 \mathrm{~s}, 60^{\circ}$ for $30 \mathrm{~s}, 72^{\circ}$ for $\left.1 \mathrm{~min}\right)$, and a melt curve stage of $\left(95^{\circ}\right.$ for $15 \mathrm{~s}, 60^{\circ}$ for $1 \mathrm{~min}$, increase of $0.3^{\circ}$ until $95^{\circ}$ ). The expression levels of genes of interest were normalized to the expression of $A C T B$ or PGK1. Primer sequences are found in Additional file 6.

Pyrosequencing of cDNA before and after DOX induction of DNA, and of clones containing integrations on alternate chromosomes were examined. Each $25 \mu \mathrm{L}$ PCR was performed with 1x PCR Buffer (Invitrogen), $0.2 \mathrm{mM}$ dNTPs, $0.625 \mathrm{U}$ Taq DNA polymerase (Invitrogen), $0.5 \mu \mathrm{M}$ forward and reverse primers, and 50-100 ng of cDNA for $(94 \mathrm{C}$ for $30 \mathrm{~s}, 58.3 \mathrm{C}$ for $30 \mathrm{~s}, 72 \mathrm{C}$ for $1 \mathrm{~min}) \times 35$ cycles, and $72{ }^{\circ} \mathrm{C}$ for $10 \mathrm{~min}$ for final extension. One of the forward and reverse primers was biotinylated for template isolation during pyrosequencing preparation. Universal M13 primer was also used for some assays (see Additional file 6), where the primer to be biotinylated instead contained the M13 sequence at the $5^{\prime}$ end (5'CGC CAG GGT TTT CCC AGT CAC GAC3'). Nested PCR was run for the assays that utilized the universal primers: the first round of PCR was performed with the same cycling conditions but only for $15 \mathrm{x}$ cycles, with the M13-tagged primer (without the biotin) and its paired forward or reverse primer; the second round of PCR was run with $1 \mu \mathrm{L}$ of PCR product from the first round of PCR as the template, as well as the biotinylated M13 primer and the non-M13-tagged primer that was used in the first round, under the same cycling condition but for $20 \mathrm{cy}-$ cles. Pyrosequencing was performed on the PyroMark MD machine (Qiagen). Template preparation for pyrosequencing was done according to manufacturer's protocol. For each assay, 10-15 $\mu \mathrm{L}$ of PCR products was used as template and CDT tips were used to dispense the dNTPs.

\section{Sequencing analysis}

For both ChIP-seq and RNA-seq, library preparation and sequencing was done according to Illumina protocols, and reads were aligned to hg18 reference genome using Tophat. RNA-seq was performed as previously described [54], first on two 8p clones following 5 days DOX and the $8 \mathrm{p}$ clone with No DOX producing $50 \mathrm{bp}$ paired-end reads, with a Pearson correlation between replicate DOX FPKM values of $r=0.9934(P<0.0001)$. A second RNA-seq of the $12 \mathrm{q}$ and $1 \mathrm{p}$ integration sites with No DOX and following 5 days DOX produced 76 bp paired-end reads. Total RNA expression was quantified in FPKM using Cufflinks. ChIP of H3K27me3 (Active Motif antibody 61017) and H3K27ac (Active Motif antibody 39133) was performed on the $8 p$ clone with and without DOX treatment, as previously done [55] using $20 \mu \mathrm{g}$ of chromatin and $3 \mu \mathrm{g}$ of antibody per IP. ChIPseq produced 36 bp single-end reads, and the ChIP-seq data were analyzed using SeqMonk to identify enriched H3K27ac peaks (MACS, $P$ value $=1 \times 10^{-3}$ ) and to quantify the level of H3K27me3 in $2 \mathrm{~kb}$ and $1 \mathrm{Mb}$ nonoverlapping windows. 
Allelic information for both RNA-seq and ChIP-seq was obtained with a custom workflow in Galaxy [56-58]. Briefly, mapped reads were run through Samtools mpileup to obtain SNPs relative to the reference genome in the control and experimental (integration of interest) samples, and allelic ratio of reads was calculated at each variant. In addition, only the variants with a biallelic ratio of $0.3-0.7$ in the No DOX (control) sample were further examined. In order to phase the variants, the allele with lower reads for each variant site in the DOX (experimental) sample was considered to be silenced in cis with the XIST transgene. Variant sites were next combined based on regions of interest, either gene location or genomic regions for RNA-seq and ChIP-seq, respectively. Variant sites that had an allelic ratio 0.15 greater or less than the overall gene allelic ratio for the sample were removed and a new overall gene ratio was calculated. For ChIP-seq, variants with reads $>2$-fold different from the average number of reads in the region of interest were also excluded. Percent allelic silencing for a given gene or region of interest was defined as (control allelic ratio experimental allelic ratio)/control allelic ratio. The calculated ratio was the frequency of the allele with lower reads in the experimental sample for each gene or region of interest. We also required that there were at least 4 reads and 5 reads with allelic information per gene (RNA-seq) and region of interest (ChIP-seq), respectively, in both the control and experimental samples to be considered for analysis.

\section{Hi-C analysis}

We obtained normalized $\mathrm{Hi}-\mathrm{C}$ data for human female fibroblast line IMR90 [45] and the $\mathrm{Hi}-\mathrm{C}$ analysis was done as previous [43]. We calculated the $1 \mathrm{Mb} \mathrm{Hi-C}$ counts by summing the counts in all $40 \mathrm{~kb}$ bins within each $1 \mathrm{Mb}$ bins across the chromosome, with the anchor being the $1 \mathrm{Mb}$ bin containing the XIST transgene (AGPAT5 for 8p clone).

\section{Data availability}

The sequence data described are available in GSE68109. Additional microscopy images are available in Figshare (http://dx.doi.org/10.6084/m9.figshare.1529822).

\section{Additional files}

Additional file 1: Genomic location of FRT integration sites in the HT1080 cell lines. (DOCX $129 \mathrm{~kb}$ )

Additional file 2: Location of XIST RNA signal for each integration site with and without DOX treatment. The numbers shown are based on three independent experiments for the DOX results and one experiment for the No DOX results, with $\geq 50$ cells counted for each integration site in each experiment. (PDF 57 kb)

Additional file 3: Candidate gene silencing assays for each integration site. One assay for each integration site was included in
Fig. 2. a Allelic pyrosequencing of three cDNAs from No DOX and DOX were compared in duplicate pyrosequencing reactions. As a control cDNA from a different integration was also assessed. b For the X-chromosome integration site $\mathrm{q}-\mathrm{RT}$-PCR was used to determine silencing as the cells are hemizygous. c Q-RT-PCR assays were also performed to validate some autosomal silencing and compare with pyrosequencing and RNA-seq. (PDF 933 kb)

Additional file 4: Imprinted regions showed broad enrichment of H3K27me3 and punctate peaks of H3K27ac. Probes with values in the extreme $5 \%$ of inputs were removed. (PDF $175 \mathrm{~kb}$ )

Additional file 5: Average $\mathrm{H} 3 \mathrm{~K} 27 \mathrm{ac}$ and $\mathrm{H} 3 \mathrm{~K} 27 \mathrm{me} 3$ for genes on $\mathbf{8 q}$ and chromosome 1. The normalized ChIP-seq level are shown across an aggregate of genes and for the $10 \mathrm{~kb}$ upstream and downstream before (NoDOX) and after XIST expression (DOX). (PDF $246 \mathrm{~kb}$ )

Additional file 6: Table of primers. (XLSX $16 \mathrm{~kb}$ )

\section{Competing interests}

The authors declare that they have no competing interests.

\section{Authors' contributions}

ADK performed the RNA FISH and IF experiments and drafted the manuscript, CY performed the RNA and ChIP-seq analysis and allelic and q-RT-PCR experiments, DCYL performed the ChIP and RNA-seq experiments, TDMD performed additional RNA FISH experiments, JM created the DS-RED construct and performed flow cytometric analyses, SELB generated and cultured the cell lines, ABB established the allelic workflow, LL and CJB provided guidance and interpretation of results and all authors contributed to the final manuscript.

\section{Acknowledgements}

This work was supported by CIHR grants to CJB (MOP-13680) and LL (MOP-119357). The authors thank Jen Chow for developing the inducible XIST clones, and Jordan Henriksen, Irene Qi and Allison M. Cotton for assistance and advice on FISH.

\section{Author details}

${ }^{1}$ Department of Medical Genetics, Molecular Epigenetics Group, Life Sciences Institute, University of British Columbia, Vancouver, Canada. ${ }^{2}$ Ludwig Institute for Cancer Research, University of California at San Diego School of Medicine, La Jolla, CA, USA. ${ }^{3}$ Division of Life Science, The Hong Kong University of Science and Technology, Clear Water Bay, Hong Kong, China.

Received: 17 April 2015 Accepted: 10 September 2015

Published online: 02 October 2015

\section{References}

1. Lyon MF. Gene action in the X-chromosome of the mouse (Mus musculus L.). Nature. 1961;190:372-3.

2. Brown CJ, Lafreniere RG, Powers VE, Sebastio G, Ballabio A, Pettigrew AL, et al. Localization of the $X$ inactivation centre on the human $X$ chromosome in Xq13. Nature. 1991;349:82-4

3. Brown CJ, Ballabio A, Rupert JL, Lafreniere RG, Grompe M, Tonlorenzi R, et al. A gene from the region of the human $X$ inactivation centre is expressed exclusively from the inactive $X$ chromosome. Nature. 1991;349:38-44.

4. Brown CJ, Hendrich BD, Rupert JL, Lafreniere RG, Xing Y, Lawrence J, et al. The human XIST gene: analysis of a $17 \mathrm{~kb}$ inactive X-specific RNA that contains conserved repeats and is highly localized within the nucleus. Cell. 1992;71:527-42

5. Clemson CM, McNeil JA, Willard HF, Lawrence JB. XIST RNA paints the inactive $X$ chromosome at interphase: evidence for a novel RNA involved in nuclear/chromosome structure. J Cell Biol. 1996;132:259-75.

6. Blewitt ME, Vickaryous NK, Hemley SJ, Ashe A, Bruxner TJ, Preis JI, et al. An $\mathrm{N}$-ethyl-N-nitrosourea screen for genes involved in variegation in the mouse. Proc Natl Acad Sci U S A. 2005;102:7629-34.

7. Pullirsch D, Hartel R, Kishimoto H, Leeb M, Steiner G, Wutz A. The Trithorax group protein Ash2l and Saf-A are recruited to the inactive $X$ chromosome at the onset of stable $X$ inactivation. Development. 2010;137:935-43. 
8. Sado T, Brockdorff N. Advances in understanding chromosome silencing by the long non-coding RNA Xist. Phil Trans Royal Society London Series B. 2013;368:20110325.

9. Barr ML, Carr DH. Correlations between sex chromatin and sex chromosomes. Acta Cytol. 1962;6:34-45.

10. Hall LL, Byron M, Sakai K, Carrel L, Willard HF, Lawrence JB. An ectopic human XIST gene can induce chromosome inactivation in postdifferentiation human HT-1080 cells. Proc Natl Acad Sci U S A. 2002;99:8677-82.

11. Chow J, Heard E. X inactivation and the complexities of silencing a sex chromosome. Curr Op Cell biol. 2009;21:359-66.

12. Wutz A, Jaenisch R. A shift from reversible to irreversible $X$ inactivation is triggered during ES cell differentiation. Mol Cell. 2000;5:695-705.

13. Rasmussen TP, Wutz A, Pehrson JR, Jaenisch R. Expression of Xist RNA is sufficient to initiate macrochromatin body formation. Chromosoma. 2001;110:411-20.

14. Csankovszki G, Panning B, Bates B, Pehrson JR, Jaenisch R. Conditional deletion of Xist disrupts histone macroH2A localization but not maintenance of X inactivation. Nat Genet. 1999;22:323-4

15. Rack KA, Chelly J, Gibbons RJ, Rider S, Benjamin D, Lafreniere RG, et al. Absence of the XIST gene from late-replicating isodicentric $X$ chromosomes in leukemia. Hum Mol Genet. 1994;3:1053-9.

16. Brown CJ, Willard HF. The human X inactivation center is not required for maintenance of X inactivation. Nature. 1994;368:154-6.

17. Agrelo R, Souabni A, Novatchkova M, Haslinger C, Leeb M, Komnenovic V, et al. SATB1 defines the developmental context for gene silencing by Xist in lymphoma and embryonic cells. Dev Cell. 2009;16:507-16.

18. Nechanitzky R, Davila A, Savarese F, Fietze S, Grosschedl R. Satb1 and Satb2 are dispensable for $X$ chromosome inactivation in mice. Dev Cell. 2012:23:866-71.

19. Jeon $Y$, Lee JT. YY1 tethers Xist RNA to the inactive $X$ nucleation center. Cell. 2011;146:119-33.

20. Chow JC, Hall LL, Baldry SE, Thorogood NP, Lawrence JB, Brown CJ. Inducible XIST-dependent X-chromosome inactivation in human somatic cells is reversible. Proc Natl Acad Sci U S A. 2007;104:10104-9.

21. Zhang LF, Huynh KD, Lee JT. Perinucleolar targeting of the inactive $X$ during $S$ phase: evidence for a role in the maintenance of silencing. Cell. 2007;129:693-706

22. Csankovszki G, Nagy A, Jaenisch R. Synergism of Xist RNA, DNA methylation, and histone hypoacetylation in maintaining $X$ chromosome inactivation. J Cell Biol. 2001;153:773-83.

23. Bhatnagar S, Zhu X, Ou J, Lin L, Chamberlain L, Zhu LJ, et al. Genetic and pharmacological reactivation of the mammalian inactive $X$ chromosome. Proc Natl Acad Sci U S A. 2014;111:12591-8.

24. Minkovsky A, Sahakyan A, Rankin-Gee E, Bonora G, Patel S, Plath K. The Mbd1-Atf7ip-Setdb1 pathway contributes to the maintenance of $X$ chromosome inactivation. Epigenetics Chromatin. 2014:7:12.

25. Yildirim E, Kirby JE, Brown DE, Mercier FE, Sadreyev RI, Scadden DT, et al. Xist RNA is a potent suppressor of hematologic cancer in mice. Cell. 2013;152:727-42.

26. Silva J, Mak W, Zvetkova I, Appanah R, Nesterova TB, Webster Z, et al. Establishment of histone h3 methylation on the inactive $\mathrm{X}$ chromosome requires transient recruitment of Eed-Enx1 polycomb group complexes. Dev Cell. 2003:4:481-95.

27. Schoeftner S, Sengupta AK, Kubicek S, Mechtler K, Spahn L, Koseki H, et al. Recruitment of PRC1 function at the initiation of $X$ inactivation independent of PRC2 and silencing. EMBO J. 2006:25:3110-22.

28. Kalantry S, Magnuson T. The Polycomb Group Protein EED Is Dispensable for the Initiation of Random X-Chromosome Inactivation. PLoS Genet. 2006;2, e66.

29. Leeb M, Wutz A. Ring1B is crucial for the regulation of developmental control genes and PRC1 proteins but not X inactivation in embryonic cells. J Cell Biol. 2007;178:219-29.

30. Tanasijevic B, Rasmussen TP. X chromosome inactivation and differentiation occur readily in ES cells doubly-deficient for macroH2A1 and macroH2A2. PLoS One. 2011;6, e21512.

31. Blewitt ME, Gendrel AV, Pang Z, Sparrow DB, Whitelaw N, Craig JM, et al. SmcHD1, containing a structural-maintenance-of-chromosomes hinge domain, has a critical role in X inactivation. Nat Genet. 2008;40:663-9.

32. Mould AW, Pang Z, Pakusch M, Tonks ID, Stark M, Carrie D, et al. Smchd regulates a subset of autosomal genes subject to monoallelic expression in addition to being critical for $X$ inactivation. Epigenetics Chromatin. 2013;6:19.

33. Chaumeil J, Le Baccon P, Wutz A, Heard E. A novel role for Xist RNA in the formation of a repressive nuclear compartment into which genes are recruited when silenced. Genes Dev. 2006:20:2223-37.
34. Thorogood NP, Brown CJ. Active chromatin marks are retained on X chromosomes lacking gene or repeat silencing despite XIST/Xist expression in somatic cell hybrids. PLoS One. 2010;5, e10787.

35. Clemson CM, Hall LL, Byron M, McNeil J, Lawrence JB. The X chromosome is organized into a gene-rich outer rim and an internal core containing silenced nongenic sequences. Proc Natl Acad Sci U S A. 2006;103:7688-93.

36. Yang C, Chapman AG, Kelsey AD, Minks J, Cotton AM, Brown CJ. X-chromosome inactivation: molecular mechanisms from the human perspective. Hum Genet. 2011;130:175-85

37. Duthie SM, Nesterova TB, Formstone EJ, Keohane AM, Turner BM, Zakian SM, et al. Xist RNA exhibits a banded localization on the inactive $X$ chromosome and is excluded from autosomal material in cis. Hum Mol Genet. 1999:8:195-204.

38. Lee JT, Strauss WM, Dausman JA, Jaenisch R. A 450 kb transgene displays properties of the mammalian X-inactivation center. Cell. 1996:86:83-94.

39. Jiang J, Jing Y, Cost GJ, Chiang JC, Kolpa HJ, Cotton AM, et al. Translating dosage compensation to trisomy 21. Nature. 2013:500:296-300.

40. Minks J, Baldry SE, Yang C, Cotton AM, Brown CJ. XIST-induced silencing of flanking genes is achieved by additive action of repeat a monomers in human somatic cells. Epigenetics Chromatin. 2013;6:23.

41. Yan C, Boyd DD. Histone $\mathrm{H3}$ acetylation and $\mathrm{H} 3 \mathrm{~K} 4$ methylation define distinct chromatin regions permissive for transgene expression. Mol Cell Biol. 2006;26:6357-71.

42. Hall LL, Carone DM, Gomez AV, Kolpa HJ, Byron M, Mehta N, et al. Stable COT-1 repeat RNA is abundant and is associated with euchromatic interphase chromosomes. Cell. 2014;156:907-19.

43. Engreitz JM, Pandya-Jones A, McDonel P, Shishkin A, Sirokman K, Surka C, et al. The Xist IncRNA exploits three-dimensional genome architecture to spread across the $X$ chromosome. Science. 2013;341:1237973.

44. Simon MD, Pinter SF, Fang R, Sarma K, Rutenberg-Schoenberg M, Bowman SK, et al. High-resolution Xist binding maps reveal two-step spreading during X-chromosome inactivation. Nature. 2013;504:465-9.

45. Dixon JR, Selvaraj S, Yue F, Kim A, Li Y, Shen Y, et al. Topological domains in mammalian genomes identified by analysis of chromatin interactions. Nature. 2012;485:376-80.

46. Nora EP, Dekker J, Heard E. Segmental folding of chromosomes: a basis for structural and regulatory chromosomal neighborhoods? BioEssays. 2013;35:818-28.

47. Lyon MF. X-chromosome inactivation: a repeat hypothesis. Cytogenet Cell Genet. 1998:80:133-7.

48. Cotton AM, Chen CY, Lam LL, Wasserman WW, Kobor MS, Brown CJ. Spread of $X$-chromosome inactivation into autosomal sequences: role for DNA elements, chromatin features and chromosomal domains. Hum Mol Genet. 2014;23:1211-23

49. Bala Tannan N, Brahmachary M, Garg P, Borel C, Alnefaie R, Watson CT, et al. DNA methylation profiling in $X$;autosome translocations supports a role for $\mathrm{L} 1$ repeats in the spread of X chromosome inactivation. Hum Mol Genet. 2014;23:1224-36

50. Gendrel AV, Heard E. Noncoding RNAs and epigenetic mechanisms during X-chromosome inactivation. Annu Rev Cell Dev Biol. 2014;30:561-80.

51. Gendrel AV, Apedaile A, Coker H, Termanis A, Zvetkova I, Godwin J, et al. Smchd1-dependent and -independent pathways determine developmental dynamics of $\mathrm{CpG}$ island methylation on the inactive $X$ chromosome. Dev Cell. 2012;23:265-79.

52. Wang Z, Willard HF, Mukherjee S, Furey TS. Evidence of influence of genomic DNA sequence on human X chromosome inactivation. PLoS Comput Biol. 2006;2, e113.

53. UCSC Genome Bioinformatics. Available at: http://genome.ucsc.edu/cgi-bin/hgBlat.

54. Xie W, Schultz Matthew D, Lister R, Hou Z, Rajagopal N, Ray P, et al. Epigenomic analysis of multilineage differentiation of human embryonic stem cells. Cell. 2013;153:1134-48.

55. Hawkins RD, Hon GC, Lee LK, Ngo Q, Lister R, Pelizzola M, et al. Distinct epigenomic landscapes of pluripotent and lineage-committed human cells. Cell Stem Cell. 2010;6:479-91.

56. Goecks J, Nekrutenko A, Taylor J. The Galaxy Team. Galaxy: a comprehensive approach for supporting accessible, reproducible, and transparent computational research in the life sciences. Genome Biol. 2010;11:R86.

57. Blankenberg D, Von Kuster G, Coraor N, Ananda G, Lazarus R, Mangan M, et al. Galaxy: a web-based genome analysis tool for experimentalists. Curr Protoc Mol Biol. 2010; Chapter 19:Unit 19.10.1-21.

58. Giardine B, Riemer C, Hardison RC, Burhans R, Elnitski L, Shah P, et al. Galaxy: a platform for interactive large-scale genome analysis. Genome Res. 2005;15:1451-5. 